not known how eyesight has improved. The authors could not rule out placebo and other bias effects.

Lancet http://doi.org/wdf (2014)

$$
\text { PHOTONICS }
$$

\section{Laser moves items long distances}

A laser beam can move matter tens of centimetres and in two directions.

Such tractor beams have been used to shift small objects very short distances. To scale this up, Wieslaw Krolikowski at the Australian National University in Canberra and his team fired a laser beam at goldcoated hollow glass spheres in air. The photons heated up the spheres, creating a temperature difference between their far and near surfaces. This generated a force that pushed the shells in the opposite direction to the beam. By changing the beam's polarization state, the team was able to stop the spheres or reverse their direction.

The authors say that the technique could be used to gather samples remotely and for other applications.

Nature Photonics http://doi.org/ wft (2014)

\section{METEOROLOGY}

\section{Tornadoes growing more clustered}

Tornadoes in the United States have been happening on fewer days since the 1970s, but more tornadoes have touched down (pictured) on those days.

The overall number of US tornadoes has not changed in recent decades. However, in

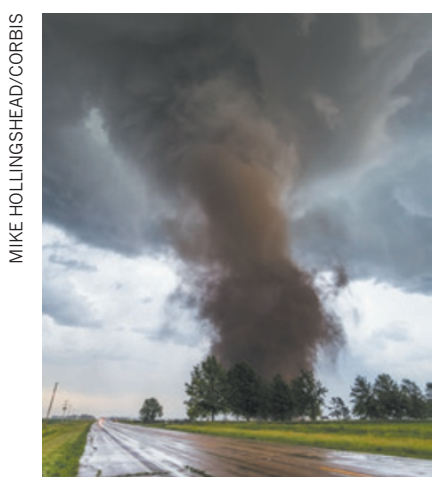

analysing the national tornado database, Harold Brooks of the National Severe Storms Laboratory in Norman, Oklahoma, and his team found that the number of days with at least one tornado has fallen from 150 to 100 since the early 1970s. Over the past decade, $20 \%$ of US tornadoes occurred on just three days of the year.

Whether the change is linked to rising global temperatures is not clear, the authors say.

Science 346, 349-352 (2014)

$$
\text { NEUROSCIENCE }
$$

\section{Molecule boosts brain rewiring}

Blocking a brain-cell receptor boosts the brain's ability to form new neuronal connections as it adapts to changing stimuli.

Carla Shatz at Stanford University in California and her colleagues disrupted the receptor, PirB, in the visual centre of mouse brains by either genetically deleting it or blocking it with a molecule.

They found that when these mice were forced to use only one eye, circuits in their visual cortices were able to rewire better than those of normal mice. This happened even in adulthood, when braincell rewiring becomes more difficult. In a mouse model of amblyopia, or 'lazy eye', the blocking molecule made the brain sensitive to signals from the unused eye, allowing better vision in that eye.

Targeting PirB could be a way to treat amblyopia and other brain disorders, the authors say.

Sci. Transl. Med. 6, 258ra140 (2014)

\section{PALAEONTOLOGY}

\section{Strange fossil is a vertebrate cousin}

Bizarre 500-million-yearold sea creatures called vetulicolians are relatives of vertebrates.

Palaeontologists have struggled to identify the relationship between living animals and these extinct

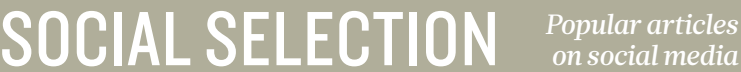

\section{Pros and cons of the PhD glut}

Amid increased competition for faculty jobs in biomedicine, some have suggested cutting the number of $\mathrm{PhD}$ students. So when a senior scientist advised against this, the online world took notice. Eve Marder, a neuroscientist at Brandeis University in Waltham, Massachusetts, argued in the journal eLife that it is hard to predict who will excel in science, so any attempt to limit access to $\mathrm{PhD}$ programmes will inevitably exclude potential stars. The reaction was mixed. "Reduce the number of admitted graduate students? Agree with Eve Marder: not the greatest idea," tweeted Sergey Kryazhimskiy, an evolutionary biologist at Harvard University in Cambridge, Massachusetts. But Mike White, a geneticist at Washington University School of Medicine in St. Louis, Missouri, argued in a blog post that Marder was "perpetuating the $\mathrm{PhD}$ pyramid scheme".

eLife 3, e04901 (2014)

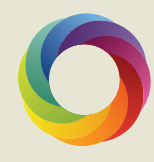

Based on data from altmetric.com. Altmetric is supported by Macmillan Science and Education, which owns Nature Publishing Group.
ONATURE.COM

For more on popular papers: go.nature.com $/ \mathrm{t} 9$ tgts organisms, because of their odd combination of features such as gill slits and a segmented abdomen. A team led by Diego García-Bellido at the University of Adelaide and John Paterson at the University of New England in Armidale, both in Australia, analysed a fossil vetulicolian from a South Australian island.

The fossil, a new species named Nesonektris aldridgei, shows the outline of a notochord - a rod-like structure that develops into the backbone in vertebrates.

Although N. aldridgei is distantly related to vertebrates, its closest relatives are tunicates - invertebrates that swim or attach themselves to underwater rocks. It was probably a free-swimming filter-feeder, say the authors. BMC Evol. Biol. 14, 214 (2014)

\section{CANCER}

\section{Immunotherapy beats leukaemia}

Engineering certain immune cells to kill cancerous cells in leukaemia has driven the disease into remission for up to two years in more than half of participants in an earlystage clinical trial.

Stephan Grupp at the Children's Hospital of Philadelphia, Pennsylvania, and his co-workers tested their approach on 30 people with acute lymphoblastic leukaemia, including 25 children, who had failed to respond to conventional treatment or relapsed.

The team engineered a patient's T cells to express a receptor that targets the cancerous B cell, and infused the T cells back into the person. After one month, 27 people were in remission, and after up to 2 years, $78 \%$ survived - a much higher rate than with chemotherapy. Those in remission had high blood levels of the engineered T cells.

However, all of the participants had inflammatory side effects that required hospitalization.

N. Engl. J. Med. 371, 1507-1517 (2014)

\section{$\rightarrow$ NATURE,COM}

For the latest research published by Naturevisit:

www.nature.com/latestresearch 